\title{
Vertical Decomposition of a Single Cell in a Three-Dimensional Arrangement of Surfaces*
}

\author{
O. Schwarzkopf ${ }^{1}$ and M. Sharir ${ }^{2}$ \\ ${ }^{1}$ Department of Computer Science, Pohang University of Science and Technology, \\ Hyoja-Dong, Pohang 790-784, South Korea \\ otfried@cs.ust.hk \\ ${ }^{2}$ School of Mathematical Sciences, Tel Aviv University, \\ Tel Aviv 69978, Israel \\ sharir@math.tau.ac.il \\ and \\ Courant Institute of Mathematical Sciences, New York University, \\ New York, NY 10012, USA
}

\begin{abstract}
Let $\Sigma$ be a collection of $n$ algebraic surface patches in $\mathbb{R}^{3}$ of constant maximum degree $b$, such that the boundary of each surface consists of a constant number of algebraic arcs, each of degree at most $b$ as well. We show that the combinatorial complexity of the vertical decomposition of a single cell in the arrangement $\mathcal{A}(\Sigma)$ is $O\left(n^{2+\varepsilon}\right)$, for any $\varepsilon>0$, where the constant of proportionality depends on $\varepsilon$ and on the maximum degree of the surfaces and of their boundaries. As an application, we obtain a near-quadratic motionplanning algorithm for general systems with three degrees of freedom.
\end{abstract}

\section{Introduction}

Let $\Sigma=\left\{\sigma_{1}, \ldots, \sigma_{n}\right\}$ be a collection of $n$ algebraic surface patches in $\mathbb{R}^{3}$ of constant maximum degree $b$, such that the boundary of each surface consists of a constant number of algebraic arcs, each of degree at most $b$ as well. Let $\mathcal{A}(\Sigma)$ denote the arrangement of $\Sigma$. (We assume that the reader is familiar with arrangements-see, for instance, the

* Work on this paper by the first author has been supported by the Netherlands' Organization for Scientific Research (NWO), by the nondirected research fund of the Korean Ministry of Education, and partial support by Pohang University of Science and Technology Grants P95015, 1995, and 96F004, 1996. Work on this paper by the second author has been supported by NSF Grants CCR-93-11127 and CCR-94-24398, by a Max-Planck Research Award, and by grants from the U.S.-Israeli Binational Science Foundation, the Israel Science Fund administered by the Israeli Academy of Sciences, and the G.I.F., the German-Israeli Foundation for Scientific Research and Development. 
recent book [19] for details concerning arrangements of surfaces in higher dimensions.) Let $\omega$ be a fixed point, not lying on any surface of $\Sigma$. We denote by $\mathcal{C}_{\omega}(\Sigma)$ the (open) three-dimensional cell of $\mathcal{A}(\Sigma)$ containing $\omega$. The combinatorial complexity of $\mathcal{C}_{\omega}(\Sigma)$ is the number of vertices, edges, and 2-faces of $\mathcal{A}(\Sigma)$ appearing on the boundary of that cell. For simplicity, we measure this complexity only by the number of vertices of the cell. It is well known that the number of all other boundary features of $\mathcal{C}_{\omega}(\Sigma)$ is proportional to the number of vertices (assuming general position-see below), plus an additive term of $O\left(n^{2}\right)$.

Recently, it has been shown that the combinatorial complexity of $\mathcal{C}_{\omega}(\Sigma)$ is $O\left(n^{2+\varepsilon}\right)$, for any $\varepsilon>0$, where the constant of proportionality depends on $\varepsilon$ and on the maximum degree $b$ of the surfaces and of their boundaries [12]. The corresponding algorithmic problem, however, of computing $\mathcal{C}_{\omega}(\Sigma)$ in near-quadratic time, has been open, with the exception of several solutions for special classes of surfaces [4], [5], [14]. The main motivation for this algorithmic problem comes from motion planning, and is explained in detail in the papers just cited, and in the recent survey paper [13]. We also note that these results extend the earlier (and somewhat simpler) analysis of the complexity of lower envelopes of surfaces in higher dimensions [11], [17].

An algorithm for constructing $\mathcal{C}_{\omega}(\Sigma)$ can be obtained using the vertical decomposition of such a cell [12]-[14]. This is a standard decomposition scheme, described in detail in several recent works [8], [9], [19], that partitions cells in arrangements of algebraic surfaces into subcells of "constant description complexity" (see below), provided the maximum degree of the surfaces is a constant.

For the sake of completeness, we also give a brief informal description of the vertical decomposition. We first assume that each surface patch in $\Sigma$ is $x y$-monotone. This can always be enforced by splitting each such patch into $O(1) x y$-monotone subpatches. In the first decomposition stage, we erect within $\mathcal{C}$ a vertical "wall" up and/or down from each edge of $\mathcal{C}$ (both surface boundary edges and intersection edges of pairs of surfaces). Each such wall consists of maximal vertical segments contained in (the closure of) $\mathcal{C}$ and passing through the points of the edge. The collection of these walls partitions $\mathcal{C}$ into subcells, each having the property that it has a unique "top" facet and a unique "bottom" facet (one or both of these facets may be undefined when the subcell is unbounded; all other facets of the subcell lie on the vertical walls). However, the complexity of each subcell may still be arbitrarily large. Thus, in the second decomposition stage, we take each subcell $\mathcal{C}^{\prime}$, project it onto the $x y$-plane, and apply to the projection a similar but two-dimensional vertical decomposition, erecting a $y$-vertical segment from each vertex of the projected subcell and from each point of local $x$-extremum on its edges. This yields a collection of trapezoidal-like subcells, and we then lift each of them vertically to 3 -space, to obtain a decomposition of $\mathcal{C}^{\prime}$ into prism-like subcells, each having "constant description complexity," meaning that each of them is a semialgebraic set defined by a constant number of polynomials of constant maximum degree (which depends on $b$ ). Repeating this second stage for all subcells $\mathcal{C}^{\prime}$ produced in the first stage, we obtain the desired vertical decomposition of $\mathcal{C}$. More details can be found elsewhere [8], [9], [19].

Using this decomposition scheme, we can then apply, for instance, a lazy randomized incremental algorithm [6] to construct the vertical decomposition of $\mathcal{C}_{\omega}(\Sigma)$, by adding the surfaces one after the other in random order, and by updating the decomposition as the surfaces are added. The efficiency of this algorithm crucially depends on the size 
(number of subcells) of the decomposition (of the cells $\mathcal{C}_{\omega}\left(\Sigma^{\prime}\right)$, for any subset $\Sigma^{\prime} \subseteq \Sigma$ ). A near-quadratic bound on the size of the vertical decomposition of a single cell implies that the (expected) complexity of the above algorithm is also near-quadratic.

In this paper we show that the complexity of the vertical decomposition of a single cell in a three-dimensional arrangement, as above, is indeed $O\left(n^{2+\varepsilon}\right)$, for any $\varepsilon>0$, where the constant of proportionality depends, as above, on $\varepsilon$ and on the maximum degree $b$ of the surfaces and of their boundaries. The proof technique borrows ideas from several recent papers [1], [11], [17], [20] that have analyzed several related problems.

It is instructive to note that if all our surfaces are $x y$-monotone without boundaries (in other words, they are graphs of continuous totally defined algebraic bivariate functions), then the near-quadratic bound on the complexity of the vertical decomposition of a single cell is an immediate consequence of the recent results of Agarwal et al. [1], which give a near-quadratic bound for the complexity of the vertical decomposition of the region enclosed between the lower envelope of one collection of such surfaces and the upper envelope of another such collection; in this special case our single cell is a portion of such a "sandwiched" region. In the general case that involves surface patches, though, the topological structure of a single cell can be much more complex, and this makes the analysis considerably harder.

As a corollary of our bound, we obtain that a single cell in a three-dimensional arrangement of surfaces, as above, can be constructed in randomized expected $O\left(n^{2+\varepsilon}\right)$ time, for any $\varepsilon>0$. This in turn implies that motion planning for fairly general systems with three degrees of freedom can be performed in near quadratic time. This solves one of the major open problems in the area. These applications of our bound are briefly presented in Section 3.

\section{Complexity of the Vertical Decomposition of a Single Cell}

Let $\Sigma$ and $\omega$ be as in the Introduction. For the purpose of our analysis, we require the surface patches to be $x y$-monotone. This involves no real loss of generality, because, as already mentioned in the Introduction, we can partition each of the surfaces into a constant number of $x y$-monotone portions (where the constant depends on the maximum degree $b$ ).

As is well known [19], the complexity of the vertical decomposition of $\mathcal{C}=\mathcal{C}_{\omega}(\Sigma)$ is proportional (up to an additive near-quadratic term) to the number of vertical visibility configurations. These are ordered triples $\left(e, e^{\prime}, g\right)$, where $e$ and $e^{\prime}$ are edges of $\mathcal{C}$ and $g$ is a vertical segment whose bottom endpoint lies on $e$, whose top endpoint lies on $e^{\prime}$, and whose relative interior is contained in $\mathcal{C}$. The main result of this paper is a near-quadratic upper bound on the number of these vertical visibility configurations.

In what follows, we assume that the surfaces $\Sigma$ are in general position, in the sense considered in Section 7.1 of [19]. This does not involve any real loss of generality. To see this, we apply a random perturbation to the polynomials defining the given surfaces. Any given vertical visibility configuration will still appear after the perturbation with some positive probability (in most cases it will actually multiply). This argument is discussed in detail in [17]. Hence, a bound for surfaces in general position will, multiplied by the appropriate constant, hold for arbitrary surfaces. 
A first consequence of the general position assumption is that the maximum number of vertical visibility configurations that correspond to any fixed pair $\left(e, e^{\prime}\right)$ of vertically visible edges is at most some constant $s$ (which depends on the maximum degree $b$ of the surfaces and of their boundaries). Therefore, it suffices to count the number of vertically visible pairs of edges of $\mathcal{C}$. These are ordered pairs $\left(e, e^{\prime}\right)$ of edges of $\mathcal{C}$ such that there exists a vertical visibility configuration $\left(e, e^{\prime}, g\right)$.

If $e$ or $e^{\prime}$ is a portion of the boundary of a surface of $\Sigma$, we call $\left(e, e^{\prime}, g\right)$ an outer vertical visibility configuration; otherwise $\left(e, e^{\prime}, g\right)$ is an inner configuration. We later show that the overall number of outer configurations is $O\left(n \lambda_{s+2}(n)\right)$, where $\lambda_{s}(n)$ is the maximum length of an $(n, s)$ Davenport-Schinzel sequence [2], [15], [19]. Hence, in what follows, we only consider inner vertical visibility configurations. For convenience, we do not mention the qualifier "inner" from now on.

For technical reasons, we extend the notion of vertical visibility configurations as follows. Let $e$ and $e^{\prime}$ be two edges of $\mathcal{A}(\Sigma)$ such that there exists a vertical segment $g$ whose bottom endpoint lies on $e$ and whose top endpoint lies on $e^{\prime}$. We say that $\left(e, e^{\prime}, g\right)$ is a vertical edge-crossing at $\mathcal{C}$-level $\xi$ if ${ }^{1}$

(i) the subset $\Sigma^{\prime} \subseteq \Sigma$ of surfaces that intersect the relative interior of $g$ has cardinality $\xi$, and

(ii) $g$ is fully contained in $\mathcal{C}_{\omega}\left(\Sigma \backslash \Sigma^{\prime}\right)$.

(The $x y$-monotonicity of the surfaces imply that $\left(\bar{e}, \bar{e}^{\prime}, g\right)$ is a vertical visibility configuration in $\mathcal{A}\left(\Sigma \backslash \Sigma^{\prime}\right)$, where $\bar{e}$ (resp. $\left.\bar{e}^{\prime}\right)$ is the edge of $\mathcal{A}\left(\Sigma \backslash \Sigma^{\prime}\right)$ containing $e$ (resp. $\left.e^{\prime}\right)$.) Note that the four surfaces incident to $e$ and $e^{\prime}$ cannot intersect the relative interior of $g$. Thus, vertical edge-crossings at $\mathcal{C}$-level 0 are precisely the vertical visibility configurations. We denote by $C_{q}(\Sigma ; \omega)$ the number of vertical edge-crossings of $\mathcal{C}$-level at most $q$. We also denote by $C_{q}(n)$ the maximum possible value of $C_{q}(\Sigma ; \omega)$, over all collections $\Sigma$ of $n$ surfaces as above (with a fixed $b$ ), and over all points $\omega$ not lying on any surface.

The notion of levels is also extended to vertices and edges of $\mathcal{A}(\Sigma)$ : We say that a vertex $v$ (resp. an edge $e$ ) of $\mathcal{A}(\Sigma)$ is at $\mathcal{C}$-level $\xi$ if there exists a subset $\Sigma^{\prime}$ of $\xi$ surfaces, so that $v$ is a vertex of (resp. $e$ is contained in an edge of) $\mathcal{C}_{\omega}\left(\Sigma \backslash \Sigma^{\prime}\right)$, and if $\xi$ is the smallest number with that property. Again, the actual vertices and edges of $\mathcal{C}_{\omega}(\Sigma)$ are precisely the vertices and edges at $\mathcal{C}$-level 0 .

Let $k$ be a threshold parameter, whose value is specified later. Our goal is to prove a bound on $C_{0}(n)$ that has roughly the form

$$
C_{0}(n) \leq \frac{1}{k^{2}} C_{k}(n)+O\left(k^{\alpha} n^{2+\varepsilon}\right)
$$

where $\alpha$ is some fixed exponent, from which we can deduce the near-quadratic bound on $C_{0}(n)$, by using Clarkson and Shor's technique [10] to bound $C_{k}(n)$ by $O\left(k^{4} C_{0}(n / k)\right)$, and by solving the resulting recurrence for $C_{0}$, which easily yields a near-quadratic upper bound. The exact inequality that we will derive will be somewhat weaker than (1), but it will still yield the desired bound on $C_{0}(n)$.

\footnotetext{
${ }^{1}$ We advise the reader that the paper uses several different notions of a level for a variety of configurations. Intuitively, any of these levels is defined to be the smallest number of surfaces whose removal causes the configuration in question to be such that all its defining elements lie on the boundary of the cell containing $\omega$.
} 
The idea of proving a bound like (1) is to identify about $k^{2} C_{0}(\Sigma ; \omega)$ distinct edgecrossings at $\mathcal{C}$-level at most $k$ in the arrangement $\mathcal{A}(\Sigma)$. We do (something close to) this, using a two-stage counting argument, similar to that used by Agarwal et al. [1]. This should be compared with the simpler technique of using a single counting stage, as used in [11] and [17] and in several other recent papers. The typical way in which such a stage is applied, say to bound the complexity of the lower envelope of surfaces in 3-space, is as follows. Start at a vertex $v$ of the envelope, and proceed from it away from the envelope along an intersection curve $\gamma$ of the surfaces, in an attempt to collect at least $k$ vertices of the arrangement along $\gamma$ before getting back to the envelope, and then charge $v$ to the first $k$ such vertices, observing that all the charged vertices are at level at most $k$, and apply the Clarkson-Shor technique to bound the number of charged vertices by $O\left(k^{3}\right)$ times the complexity of the envelope of a sample of $n / k$ of the surfaces. This approach, combined with many additional ingredients that we omit in this sketchy review, leads to a recurrence that solves to $O\left(n^{2+\varepsilon}\right)$, for any $\varepsilon>0$, which is smaller by about one factor of $n$ than the naive bound $O\left(n^{3}\right)$.

In contrast, the naive bound on the number of edge-crossings is $O\left(n^{4}\right)$, since each crossing is determined by four surfaces, and we want to obtain a bound that is about two factors of $n$ smaller. This is why we need a two-stage counting scheme. Unfortunately, such a scheme is considerably more complex, and we discuss below the additional complications that may arise, and give some intuitive explanation of the manner in which we overcome them, before giving the full formal proof.

Preliminaries. Let $e$ be an edge of $\mathcal{A}(\Sigma)$ and let $V_{e}$ be the vertical 2-manifold obtained as the union of all $z$-vertical rays whose bottom endpoints lie on $e$. The intersection of each surface $\sigma \in \Sigma$ with $V_{e}$ is a (not necessarily connected) algebraic arc of constant maximum degree (and with a constant number of connected components), so each pair of these arcs intersect in at most some constant number, $s$, of points (where $s$ depends only on the maximum degree $b$ of the given surfaces and of their boundaries; it is the same parameter $s$ mentioned at the beginning of this section). We denote the set of these arcs by $\Sigma_{e}$, and their arrangement on $V_{e}$ by $\mathcal{A}\left(\Sigma_{e}\right)$.

Completely analogously, we define the vertical 2-manifold $V^{e}$ obtained as the union of all downward directed $z$-vertical rays whose top endpoints lie on $e$. (Imagine $V^{e}$ as a "curtain" hanging down from $e$, while $V_{e}$ is a curtain standing on $e$.) We denote the set of arcs formed by the intersections of the surfaces of $\Sigma$ with $V^{e}$ by $\Sigma^{e}$, and their arrangement in $V^{e}$ by $\mathcal{A}\left(\Sigma^{e}\right)$. We define the $V$-level of a point $p$ in $V_{e}$ (resp. in $V^{e}$ ) to be the number of arcs in $\Sigma_{e}$ (resp. in $\Sigma^{e}$ ) that lie below (resp. above) $p$.

A simple but crucial observation is:

Lemma 2.1. Let e be an edge of $\mathcal{A}(\Sigma)$ with $\mathcal{C}$ lying locally right above e. Then $\left(e, e^{\prime}, g\right)$ is an edge-crossing at $\mathcal{C}$-level $\xi$, if and only if the point of $e^{\prime} \cap V_{e}$ that lies on the z-vertical line through $g$ is a vertex of $\mathcal{A}\left(\Sigma_{e}\right)$ at $V$-level $\xi$.

See Fig. 1 for an illustration. This lemma implies that each vertical visibility configuration with bottom edge $e$ corresponds to a vertex in the lower envelope of the arcs in $\Sigma_{e}$. (Of course, a similar and symmetric statement holds for $\Sigma^{e^{\prime}}$, where $e^{\prime}$ is the top edge of the configuration.) 


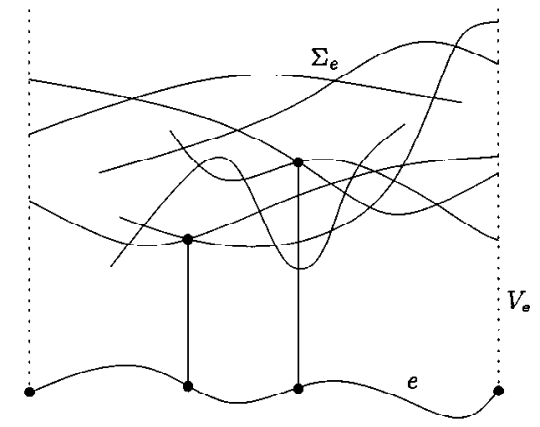

Fig. 1. The arrangement $\mathcal{A}\left(\Sigma_{e}\right)$; with vertices representing a vertical visibility configuration and a vertical edge-crossing at $\mathcal{C}$-level 3 , both having $e$ as their bottom edge.

Now that we have introduced this terminology and observations, we can dispose, as promised, of outer visibility configurations:

Lemma 2.2. The number of outer vertical visibility configurations is $O\left(n \lambda_{s+2}(n)\right)$.

Proof. Let $\delta$ be an arc bounding some surface in $\Sigma$. By Lemma 2.1 each outer vertical visibility configuration having $\delta$ as its bottom edge is represented by some vertex (breakpoint) of the lower envelope of $\Sigma_{\delta}$ within $V_{\delta}$. By the standard Davenport-Schinzel theory [2], [15], [19], the number of such breakpoints is $O\left(\lambda_{s+2}(n)\right)$ (recall that $\Sigma_{\delta}$ consists of $O(n)$ connected arcs, each pair of which intersect in at most $s$ points). We repeat this analysis for each of the $O(n)$ boundary arcs of the surfaces of $\Sigma$, and also apply a symmetric analysis within the "hanging curtains" $V^{\delta}$. This implies the assertion of the lemma.

It is convenient for our analysis to assume that the arrangements $\mathcal{A}\left(\Sigma_{e}\right)$ and $\mathcal{A}\left(\Sigma^{e^{\prime}}\right)$ do not contain any arc endpoints at $V$-level $\leq 3 k$, except on the relative boundaries of $V_{e}$ and $V^{e^{\prime}}$ (recall that all other arc endpoints lie on surface boundaries). Intuitively, the reason for this is that later in the analysis we will need to argue that many arcs that appear among the first $k$ or $2 k V$-levels in $V_{e}$ or $V^{e^{\prime}}$ contain at least $k$ vertices of the corresponding arrangement. The above requirement guarantees that all but at most $3 k$ such arcs have the desired property. More details are given later in the proof. We can achieve this by splitting the edges of $\mathcal{A}(\Sigma)$ into what we call split edges, as follows.

Let $\gamma$ be an arc in $\Sigma_{e}$. If $\gamma$ has an endpoint $w$ within the relative interior of $V_{e}$, which lies in the first $3 k V$-levels of $\mathcal{A}\left(\Sigma_{e}\right)$, we erect a vertical line through $w$ and split $e$ and $V_{e}$ at that line into two portions. We repeat this splitting for all edges $e$ of $\mathcal{A}(\Sigma)$ and for each $\gamma \in \Sigma_{e}$, whenever it is applicable. We apply a symmetric procedure in all the corresponding downward-directed curtains $V^{e^{\prime}}$. Furthermore, we split all edges $e$ at points where their projection onto the $x y$-plane has a tangent parallel to the $y$-axis. This will guarantee that all split edges are $x$-monotone.

Lemma 2.3. The overall number of such edge-splittings is $O\left(k^{2} n \lambda_{s+2}(n / k)\right)$. 


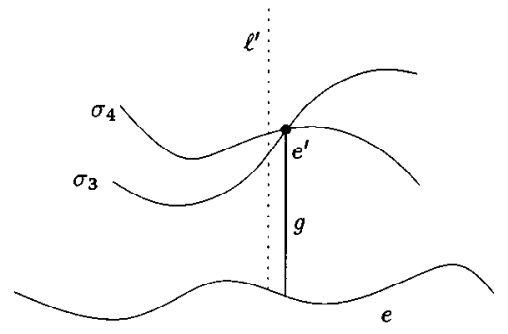

Fig. 2. An edge-crossing $\chi=\left(e, e^{\prime}, g\right)$ with $\sigma(\chi)=\sigma_{3}$.

Proof. The intersection curve of two surfaces has only a constant number of points where the projection on the $x y$-plane has a tangent parallel to the $y$-axis, so the total number of such points is $O\left(n^{2}\right)$.

We bound the number of splits induced by an endpoint at $V$-level at most $3 k$ using a similar argument to that of Lemma 2.2. Let $\delta$ be an arc bounding some surface in $\Sigma$. It is easily seen that each edge-split induced by $\delta$ (i.e., a splitting of some edge $e$ caused by a point in $\delta \cap V_{e}$ (or a point in $\delta \cap V^{e}$ ) that lies at $V$-level $\leq 3 k$ in $\mathcal{A}\left(\Sigma_{e}\right.$ ) (or in $\mathcal{A}\left(\Sigma^{e}\right)$ )) corresponds to a vertex of $\mathcal{A}\left(\Sigma_{\delta}\right)$ in $V_{\delta}$, or to a vertex of $\mathcal{A}\left(\Sigma^{\delta}\right)$ in $V^{\delta}$, at $V$-level $\leq 3 k$, and a standard application of the Clarkson-Shor technique implies that the overall number of such vertices, over all boundary arcs $\delta$, is $O\left(k^{2} n \lambda_{s+2}(n / k)\right)$ [10], [16].

We make one final definition before we start with the actual proof. For a vertical edge-crossing $\chi=\left(e, e^{\prime}, g\right)$, we have four distinct surfaces $\sigma_{1}, \sigma_{2}, \sigma_{3}, \sigma_{4} \in \Sigma$, such that $e \subset \sigma_{1} \cap \sigma_{2}$ and $e^{\prime} \subset \sigma_{3} \cap \sigma_{4}$ (here we already assume that $e$ and $e^{\prime}$ are split edges). Let $\ell$ be the vertical line through $g$, and let $\ell^{\prime}$ be a copy of $\ell$ shifted infinitesimally along $e$ in decreasing $x$-direction. Then $\sigma_{3} \cap \ell^{\prime}$ and $\sigma_{4} \cap \ell^{\prime}$ are two distinct points. We put $\sigma(\chi)=\sigma_{3}$ if $\sigma_{3} \cap \ell^{\prime}$ lies below $\sigma_{4} \cap \ell^{\prime}$, otherwise $\sigma(\chi)=\sigma_{4}$; see Fig. 2 .

Informal Preview of the Proof. As mentioned above, the proof uses a two-stage argument. The first stage analyzes the structure of the arrangements $\mathcal{A}\left(\Sigma_{e}\right)$, for (split) edges $e$ of the given cell, and identifies within them a set $\mathcal{R}$ of edge-crossings, all at $\mathcal{C}$-level at most $k$, such that $C_{0}(\Sigma ; \omega)$ is upper bounded by roughly $O(|\mathcal{R}| / k)$ plus near-quadratic terms. The second stage then analyzes the number of edge-crossings in $\mathcal{R}$, by "distributing" them over the arrangements $\mathcal{A}\left(\Sigma^{e^{\prime}}\right)$, over all (split) edges $e^{\prime}$ at $\mathcal{C}$-level at most $k$, and by estimating their number within each such arrangement. The goal is to show that $|\mathcal{R}|$ is upper bounded by roughly $O\left(C_{3 k}(n) / k\right)$ plus certain near-quadratic terms. To do so, we want to argue that the number of vertices of the first $3 k V$-levels in any such arrangement is at least roughly $\Omega(k)$ times the number of vertices representing elements of $\mathcal{R}$ in that arrangement. This argument, however, is complicated by the fact that some arrangements $\mathcal{A}\left(\Sigma^{e^{\prime}}\right)$ may contain too many of the edge-crossings in $\mathcal{R}$. To overcome this problem, we classify the crossings in $\mathcal{R}$ into two categories that we call "covered" and "uncovered." In two central lemmas (Lemmas 2.5 and 2.6) we then show that the above argument holds for the number of uncovered edge-crossings in $\mathcal{R}$, and that the 
number of covered edge-crossings is near quadratic, which follows from a somewhat involved graph planarity argument. Putting everything together, we obtain a recurrence formula that solves to the desired near-quadratic bound for $C_{0}(n)$.

First Stage. In this stage we identify a set $\mathcal{R}$ of special vertical edge-crossings in $\mathcal{A}(\Sigma)$.

Consider first a (split) edge $e$ of $\mathcal{C}$ with $\mathcal{C}$ lying locally right above $e$. We partition $e$ into two subedges as follows: We start from the right endpoint of $e$ (recall that all split edges are $x$-monotone) and move along $e$ to the left until we encounter the $(k+1)$ st distinct surface of $\Sigma$ directly above the point. We denote the portion of $e$ traversed by this process by $e_{r}$, and the remaining part of $e$ by $e_{l}$. (It can happen that we encounter the left endpoint of $e$ before seeing more than $k$ distinct surfaces-in that case $e_{r}=e$ and $e_{l}$ is empty.) By Lemma 2.1, every edge-crossing at $\mathcal{C}$-level 0 with bottom point on $e_{r}$ corresponds to a vertex of the lower envelope of $\mathcal{A}\left(\Sigma_{e_{r}}\right)$ on $V_{e_{r}}$. Since there are only $k$ surfaces appearing on the lower envelope over $e_{r}$, its complexity is at most $O\left(\lambda_{s+2}(k)\right)$ [2], [15]. Since the number of edges bounding $\mathcal{C}$ is $O\left(n^{2+\varepsilon}\right)$ [12], and they can be split into at most $O\left(k^{2} n \lambda_{s+2}(n / k)\right)$ additional split edges, the overall number of vertical visibility configurations involving the right subedges $e_{r}$ of all the split edges $e$ of $\mathcal{C}$ is at most $O\left(\lambda_{s+2}(k)\left(n^{2+\varepsilon}+k^{2} n \lambda_{s+2}(n / k)\right)\right)$, for any $\varepsilon>0$. In the following, we therefore restrict our attention to the vertical visibility configurations that appear above the left subedges $e_{l}$ of the (split) edges of $\mathcal{C}$.

Intuitively, the reason for considering the right portions of edges separately lies in the construction of $\mathcal{R}$, described below. In this construction we move to the right from certain vertices $v$ of the lower envelope of $\mathcal{A}\left(\Sigma_{e}\right)$ along an incident arc, in an attempt to collect at least $k$ vertices of $\mathcal{A}\left(\Sigma_{e}\right)$ along that arc, each incident to a new distinct surface. We can guarantee that this will be possible if at least $k$ distinct surfaces appear to the right of $v$ on the envelope, that is, if $v$ lies above the left portion of $e$.

Consider a pair of surfaces $\sigma_{1}, \sigma_{2} \in \Sigma$, and consider their intersection curve $\sigma_{1} \cap \sigma_{2}$. This curve consists of a constant number of $x$-monotone connected pieces. Let $\gamma$ be one such piece. Let $V_{\gamma}$ be the union of all $V_{e}$, for all (split) edges $e$ of the arrangement that are contained in $\gamma$. Let $\gamma^{\prime}$ be the subset of $\gamma$ that is the union of the left subedges $e_{l}$ of all edges $e \subseteq \gamma$ of $\mathcal{C}$ such that $\mathcal{C}$ lies locally right above $e$. Let $\Sigma(\gamma)$ be the set of surfaces in $\Sigma$ that appear on the lower envelope on $V_{\gamma}$ restricted over $\gamma^{\prime}$, and let $t=t_{\gamma}=|\Sigma(\gamma)|$. The number of breakpoints of the lower envelope above $\gamma^{\prime}$ (excluding the endpoints of $\operatorname{arcs}$ in $\left.\gamma^{\prime}\right)$ is at most $a \lambda_{s+2}(t)$ [2], [15], where $a$ is an appropriate constant (depending on the maximum degree of the surfaces; it arises because, as above, an intersection $\sigma \cap V_{\gamma}$ may consist of more than one connected arc).

Consider now a surface $\sigma \in \Sigma(\gamma)$. It appears on the lower envelope over the left subedge of some (split) edge $e \subseteq \gamma$ with $\mathcal{C}$ lying locally right above $e$, and therefore there are at least $k$ surfaces $\sigma^{\prime} \in \Sigma$ that appear over $e$ to the right of $\sigma$. By continuity and by our construction, either $\sigma$ and such a surface $\sigma^{\prime}$ intersect within $V_{e}$ at least once, or each of them has a point at $V$-level $>k$. We now collect $k$ vertices on $\sigma \cap V_{e}$ as follows: We start at some point where $\sigma$ appears on the lower envelope on $V_{e}$ (over the left subedge $e_{l}$ ), and follow $\sigma \cap V_{e}$ in increasing $x$-direction (recall that all split edges are $x$-monotone). We pass, before we reach the end of $e$, at least $k$ vertices $v$, at which we encounter a new, distinct surface in $\Sigma$, because we must either encounter all the $k$ surfaces that appear above $e_{r}$ or reach the $k$ th $V$-level. All these vertices are at 


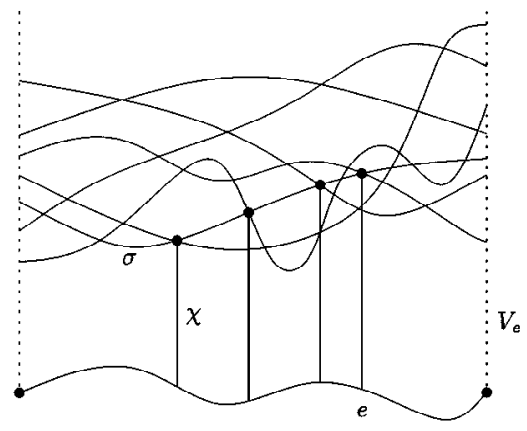

Fig. 3. The setup in the construction of $\mathcal{R}$.

$V$-level $\leq k$, since when we first reach the $k$ th $V$-level, we must have passed all the $k$ surfaces lying below the point. For every such vertex $v$, let $\chi_{v}$ be the vertical edgecrossing with bottom edge $e$ corresponding to $v$ by Lemma 2.1. Note that $\sigma\left(\chi_{v}\right)=\sigma$. See Fig. 3 for an illustration. We let $\mathcal{R}(\gamma, \sigma)$ denote the collection of these edge-crossings $\chi_{v}$. Note that we collect these $k$ vertices starting from only one occurrence of $\sigma$ along the entire curve $\gamma$, so $|\mathcal{R}(\gamma, \sigma)|=k$. This is used in deriving property (R3) of Lemma 2.4 below. Put $\mathcal{R}(\gamma)=\bigcup_{\sigma \in \Sigma(\gamma)} \mathcal{R}(\gamma, \sigma)$, and $\mathcal{R}=\bigcup_{\gamma} \mathcal{R}(\gamma)$, over all $x$-monotone pieces $\gamma$ of intersection curves. The fact that $\sigma\left(\chi_{v}\right)=\sigma$ for all $\chi_{v} \in \mathcal{R}(\gamma, \sigma)$ implies that the sets $\mathcal{R}(\gamma, \sigma)$ are pairwise disjoint.

As observed before, the number of visibility configurations above $\gamma^{\prime}$ is at most $a \lambda_{s+2}(t)$. On the other hand, we have that

$$
|\mathcal{R}(\gamma)|=\left|\bigcup_{\sigma \in \Sigma(\gamma)} \mathcal{R}(\gamma, \sigma)\right|=k t=\Omega\left(k \frac{t}{\lambda_{s+2}(t)} \cdot a \lambda_{s+2}(t)\right) \geq \frac{k}{\beta(n)} \cdot a \lambda_{s+2}\left(t_{\gamma}\right),
$$

where $\beta(n)=\Theta\left(\lambda_{s+2}(n) / n\right)$ is an extremely slowly growing function of $n$ [2], [15]. Summing (2) over all $x$-monotone pieces $\gamma$ of intersection curves, and observing that any edge-crossing in $\mathcal{R}$ is counted in this sum exactly once, we obtain

$$
C_{0}(\Sigma ; \omega) \leq \frac{\beta(n)}{k}|\mathcal{R}|+O\left(\lambda_{s+2}(k)\left(n^{2+\varepsilon}+k^{2} n \lambda_{s+2}\left(\frac{n}{k}\right)\right)\right),
$$

for any $\varepsilon>0$ (where the second term in the right-hand side bounds the number of vertical visibility configurations over the right portions $e_{r}$ ). We thus obtain the following lemma.

Lemma 2.4. Given a set of surfaces $\Sigma$ and a point $\omega$ as above, there is a set $\mathcal{R}$ of vertical edge-crossings such that $|\mathcal{R}|$ satisfies (3) and such that the following conditions hold:

(R1) Each $\chi \in \mathcal{R}$ is at $\mathcal{C}$-level at most $k$.

(R2) For each $\chi=\left(e, e^{\prime}, g\right) \in \mathcal{R}$, the edge $e$ is an edge of $\mathcal{C}$ such that $\mathcal{C}$ lies locally right above $e$. 


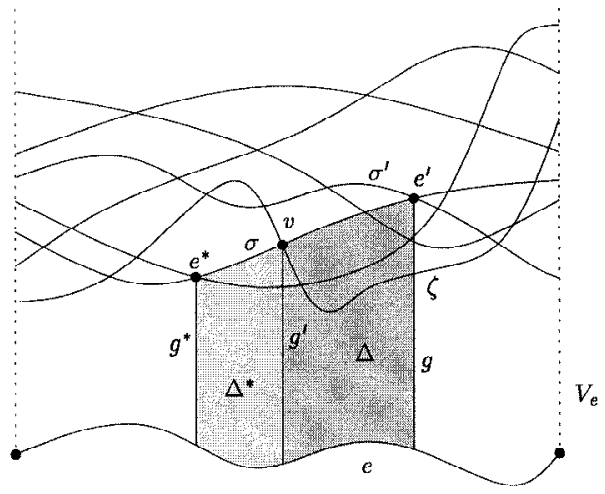

Fig. 4. Condition (R4).

(R3) For any three surfaces $\sigma_{1}, \sigma_{2}, \sigma_{3} \in \Sigma$, there are at most $\mu k$ vertical edgecrossings $\chi=\left(e, e^{\prime}, g\right)$ in $\mathcal{R}$ with $e \subseteq \sigma_{1} \cap \sigma_{2}$ and $\sigma(\chi)=\sigma_{3}$, for some constant $\mu$ depending on the maximum degree $b$.

(R4) For each $\chi=\left(e, e^{\prime}, g\right) \in \mathcal{R}$, and for any surface $\zeta$ that intersects the relative interior of $g$, there is an intersection point $v$ of $\zeta$ and $\sigma=\sigma(\chi)$ on $V_{e}$, so that the portion of $\zeta \cap V_{e}$ between $v$ and $g$ does not meet $\sigma$, and the portion of $\sigma \cap V_{e}$ between $v$ and $g$ does not meet the other surface $\sigma^{\prime}$ incident to the top endpoint of $g$. In addition, if $g^{\prime}$ denotes the vertical segment connecting $e$ and $v$, then the "trapezoidal-like" region $\Delta$ formed within $V_{e}$ by $g, g^{\prime}$, the part of e between $g$ and $g^{\prime}$, and the part of $\sigma \cap V_{e}$ between $g$ and $g^{\prime}$, is intersected by at most $k$ surfaces of $\Sigma$ (see Fig. 4).

Proof. Conditions (R1) and (R2) are immediate from the construction. Condition (R3) follows if we choose $\mu$ to be the maximum number of maximal $x$-monotone pieces into which any intersection curve between two surfaces in $\Sigma$ is decomposed. All that remains to be shown is condition (R4): Since $\chi \in \mathcal{R}$ and $\sigma(\chi)=\sigma$, there exists an edge-crossing at $\mathcal{C}$-level 0 of the form $\left(e, e^{*}, g^{*}\right)$, where $e^{*}$ is incident to $\sigma$, such that, if we follow $\sigma \cap V_{e}$ from the top endpoint of $g^{*}$ to the top endpoint of $g$, we encounter at most $k-1$ distinct surfaces of $\Sigma$, and do not encounter $\sigma^{\prime}$. Let $\Delta^{*}$ denote the trapezoidal-like region formed within $V_{e}$ by $g, g^{*}$, the part of $e$ between $g$ and $g^{*}$, and the part $\zeta$ of $\sigma \cap V_{e}$ between $g$ and $g^{*}$. Then $\Delta^{*}$ can be intersected by at most $k$ surfaces of $\Sigma$. To see this, we note that, as just argued, at most $k$ surfaces intersect $\zeta$, and that no surface can intersect $e$ (which is a portion of an edge of $\mathcal{A}(\Sigma)$ ) or $g^{*}$. We claim that no boundary arc of any surface of $\Sigma$ can cross $\Delta^{*}$. Indeed, let $u$ be the leftmost point of intersection of a boundary arc with $\Delta^{*}$. Then the number of surfaces that vertically separate $u$ from $e$ is at most $k$ (any such surface must cross $\zeta$ ), so that, by construction, such a crossing would have caused $e$ to be split below it. It now follows that any surface crossing $\Delta^{*}$ must also cross $\zeta$, and condition (R4) is thus immediate.

Second Stage. We next bound $|\mathcal{R}|$ in terms of $C_{k}(n)$. Let $e^{\prime}$ be a split edge of $\mathcal{A}(\Sigma)$ 
at $\mathcal{C}$-level $\leq k$. Let $t=t_{e^{\prime}}$ denote the number of surfaces of $\Sigma$ that appear on the first $2 k$ (top) $V$-levels of $\mathcal{A}\left(\Sigma^{e^{\prime}}\right)$. (We remind the reader that $V$-levels in $V^{e^{\prime}}$ are measured from $e^{\prime}$ downward.) If $t \leq 4 k$, then $e^{\prime}$ contributes at most $O\left(k^{2}\right)$ edge-crossings to $\mathcal{R}$. We claim that there are only $O\left(k^{1-\varepsilon} n^{2+\varepsilon}\right)$ split edges $e^{\prime}$ of $\mathcal{A}(\Sigma)$ at $\mathcal{C}$-level $\leq k$. To see this, charge each such edge $e^{\prime}$ to one of its endpoints, and observe that the endpoint is either a vertex of $\mathcal{A}(\Sigma)$ at $\mathcal{C}$-level at most $k$ or a splitting point of the edge of $\mathcal{A}(\Sigma)$ containing $e^{\prime}$. By the analysis in [10], [16], and [19], the number of endpoints of the first type is $O\left(k^{1-\varepsilon} n^{2+\varepsilon}\right)$, for any $\varepsilon>0$. The number of endpoints of the second type is, by Lemma 2.3, $O\left(k^{2} n \lambda_{s+2}(n / k)\right)$, which is subsumed by the first bound. Hence, the overall number of edge-crossings in $\mathcal{R}$ within all the curtains $V^{e^{\prime}}$ for which $t_{e^{\prime}} \leq 4 k$, is $O\left(k^{2} \cdot k^{1-\varepsilon} n^{2+\varepsilon}\right)=O\left(k^{3} n^{2+\varepsilon}\right)$, for any $\varepsilon>0$. We can thus assume that $t>4 k$.

We want to repeat, within $V^{e^{\prime}}$, the analysis of the first step. However, we face a complication that the number of edge-crossings that are counted in $\mathcal{R}$ within $V^{e^{\prime}}$ could be as large as $\Omega(t k)$, as it is possible that all vertices of $V$-level $\leq k$ in $\mathcal{A}\left(\Sigma^{e^{\prime}}\right)$ correspond to edge-crossings in $\mathcal{R}$. Our goal in this second stage is to bound $|\mathcal{R}|$ by something close to $(1 / k) C_{k}(n)$, but the technique of the first counting stage will not imply this when $V^{e^{\prime}}$ is "full" of edge-crossings in $\mathcal{R}$. To overcome this problem, we first bound the number of "excessive" edge-crossings in $\mathcal{R}$ within $V^{e^{\prime}}$, using a different approach, and only then bound the number of remaining crossings, using an approach similar to that of the first stage.

We first identify a class of vertical edge-crossings in $\mathcal{R}$ (the "excessive" edgecrossings), whose number we will be able to bound independently. We define an edgecrossing $\left(e, e^{\prime}, g\right)$ at $\mathcal{C}$-level at most $k$ to be covered if it satisfies the following condition:

Let $\sigma_{1}, \sigma_{2} \in \Sigma$ be the two surfaces incident to $e$. There is a surface $\zeta \in \Sigma$ that intersects the relative interior of $g$, and either $\sigma_{1}$ or $\sigma_{2}$ (say, for definiteness, $\sigma_{1}$ ) crosses $\zeta$ within $V^{e^{\prime}}$, either to the left or to the right of $g$, at some point $w$. Moreover, if $g^{*}$ denotes the vertical segment connecting $w$ to $e^{\prime}$, then the trapezoidal-like portion of $V^{e^{\prime}}$ bounded by $g, g^{*}$, the portion of $e^{\prime}$ between $g$ and $g^{*}$, and the portion of $\sigma_{1}$ between $g$ and $g^{*}$, is crossed by at most $2 k$ surfaces of $\Sigma$.

See Fig. 5 for an illustration of this definition; note that the definition encompasses several different subcases, as is illustrated in the figure. Note also that in the rightmost subcase shown there the intersection $w^{\prime}$ between $\zeta$ and $\sigma_{2}$ in $V^{e^{\prime}}$ could also be considered. However, the corresponding trapezoidal-like region is not properly contained in the one
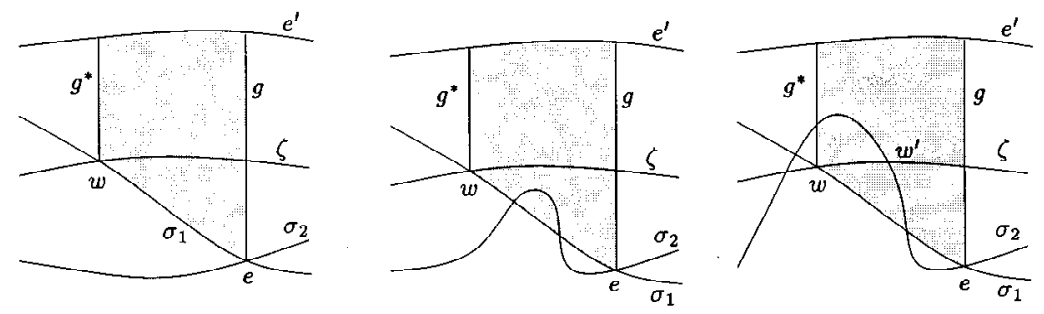

Fig. 5. Several types of covered edge-crossings. 
corresponding to $w$, so it is possible that it is crossed by more than $2 k$ surfaces and thus disqualifies for making our edge-crossing covered.

We can now establish the two central lemmas.

Lemma 2.5. The number of uncovered edge-crossings in $\mathcal{R}$ within $V^{e^{\prime}}$ is $O\left(t_{e^{\prime}} \beta^{2}\left(t_{e^{\prime}}\right)\right)$.

Proof. In the first step of the proof, we partition the arcs of $\Sigma^{e^{\prime}}$ into "small" subarcs, as follows. Recall that, by construction, each endpoint of any arc in $\Sigma^{e^{\prime}}$ must either lie on the relative boundary of $V^{e^{\prime}}$, or else be at $V$-level $>3 k$. Replace $\Sigma^{e^{\prime}}$ by the subcollection $\Sigma^{*}$ of only those $t=t_{e^{\prime}}$ arcs that appear within the first $2 k V$-levels of $\mathcal{A}\left(\Sigma^{e^{\prime}}\right)$. The total number of vertices of $\mathcal{A}\left(\Sigma^{*}\right)$ at $V$-level $\leq 2 k$ is $O\left(k^{2} \lambda_{s+2}(t / k)\right)$ [16], [19], and so there must exist a $V$-level $k \leq k^{*} \leq 2 k$ that contains only $O\left(k \lambda_{s+2}(t / k)\right)=O(t \beta(t / k))$ vertices. Hence, the portion $\mathcal{A}^{+}\left(\Sigma^{*}\right)$ of $\mathcal{A}\left(\Sigma^{*}\right)$ that lies at $V$-level $\leq k^{*}$ is formed by $O(t \beta(t / k))$ connected subarcs whose endpoints lie either on the $\left(k^{*}\right)$ th $V$-level or on the vertical boundaries of $V^{e^{\prime}}$; see Fig. 6. Moreover, since the number of vertices in $\mathcal{A}^{+}\left(\Sigma^{*}\right)$ is $O(k t \beta(t / k))$, we can partition further each of these subarcs (at points not coinciding with any vertex of the arrangement) into smaller connected pieces, so that each piece is incident to at most $k$ vertices of $\mathcal{A}^{+}\left(\Sigma^{*}\right)$, and so that the overall number of these smaller subarcs is still $O(t \beta(t / k))$; see Fig. 6. Let $\Sigma^{+}$denote the resulting collection of the new subarcs. Note that all vertices of $\mathcal{A}\left(\Sigma^{e^{\prime}}\right)$ at $V$-level $\leq k^{*}$ are also vertices of $\mathcal{A}\left(\Sigma^{+}\right)$.

Define the depth $D(\delta)$ of a subarc $\delta \in \Sigma^{+}$to be the smallest $V$-level in $\mathcal{A}\left(\Sigma^{+}\right)$of any point on $\delta$; this notion is illustrated in Fig. 6 . Note that, by construction, $D(\delta)$ is always at most $k^{*}$, and it is also equal to the smallest $V$-level in $\mathcal{A}\left(\Sigma^{e^{\prime}}\right)$ of any point on $\delta$. We have:

Claim. Let $\left(e, e^{\prime}, g\right)$ be an uncovered edge-crossing in $\mathcal{R}$ within $V^{e^{\prime}}$, and suppose that the relative interior of $g$ is crossed by $h \leq k$ surfaces. Let $\sigma_{1}$ and $\sigma_{2}$ be the two surfaces incident to $e$, and let $\delta_{1}, \delta_{2} \in \Sigma^{+}$be the respective subarcs of $\sigma_{1} \cap V^{e^{\prime}}, \sigma_{2} \cap V^{e^{\prime}}$ incident to the bottom endpoint $v$ of $g$. Then $D\left(\delta_{1}\right)=D\left(\delta_{2}\right)=h$.

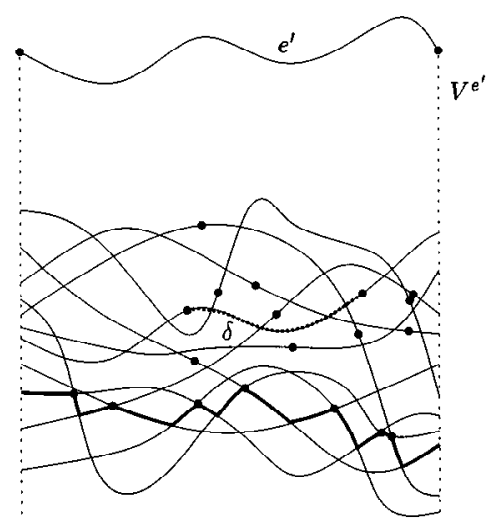

Fig. 6. Clipping the first $k^{*} V$-levels of $\mathcal{A}\left(\Sigma^{e^{\prime}}\right)$ and decomposing them into subarcs; the depth of the subarc $\delta$ is 2 . 


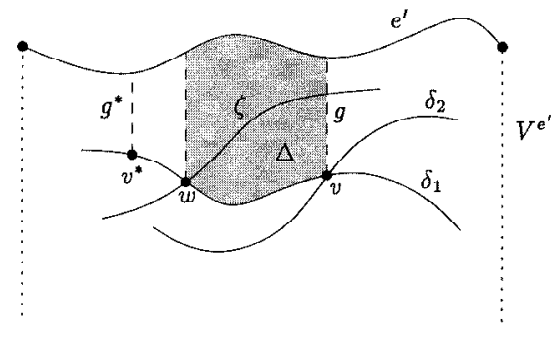

Fig. 7. The proof of the bound on the number of uncovered edge-crossings.

Proof of Claim. Note that, by definition, both $D\left(\delta_{1}\right)$ and $D\left(\delta_{2}\right)$ are at most $h$. Suppose to the contrary that, say, $D\left(\delta_{1}\right)<h$. Then there is a point $v^{*} \in \delta_{1}$ whose $V$-level $h^{*}$ is strictly smaller than $h$; see Fig. 7. This implies that one of the surfaces, call it $\zeta$, that crosses the relative interior of $g$ cannot cross the relative interior of the vertical segment $g^{*}$ that connects $v^{*}$ to $e^{\prime}$. However, then $\zeta$ must cross $\delta_{1}$ at some point $w$ between $v$ and $v^{*}\left(\zeta\right.$ cannot cross $e^{\prime}$ and since $\delta_{1}$ has no point of $V$-level $>k^{*}$, there cannot be a boundary point above $\delta_{1}$ within $\left.V^{e^{\prime}}\right)$. It is now easy to see that $\left(e, e^{\prime}, g\right)$ is a covered edge-crossing. Indeed, let $\Delta$ denote the trapezoidal-like region formed within $V^{e^{\prime}}$ by $g$, the vertical segment connecting $w$ to $e^{\prime}$, the portion of $\delta_{1}$ between $v$ and $w$, and $e^{\prime}$. Any surface of $\Sigma$ intersecting $\Delta$ must either intersect the interior of $g$ or must form a vertex on $\delta_{1}$ between $v$ and $w$. There are at most $k$ surfaces of the first kind, and at most $k-2$ of the second (recall that $\delta_{1}$ has at most $k$ vertices in $\mathcal{A}\left(\Sigma^{e^{\prime}}\right)$ ), and so $\Delta$ is crossed by less than $2 k$ surfaces. This contradiction completes the proof of the claim.

Let $\Sigma_{h}^{+}$denote the set of all subarcs of $\Sigma^{+}$whose depth is $h$, for $h=1, \ldots, k$, and put $t_{h}=\left|\Sigma_{h}^{+}\right|$. If $\left(e, e^{\prime}, g\right)$ is as in the claim, then the corresponding vertex $v$ is a vertex of the upper envelope of $\Sigma_{h}^{+}$within $V^{e^{\prime}}$ : Indeed, the claim implies that $v$ is a vertex of $\mathcal{A}\left(\Sigma_{h}^{+}\right)$, and no subarc of $\Sigma_{h}^{+}$can pass above $v$, because the depth of any such subarc must be strictly smaller than $h$. Hence, the number of uncovered edge-crossings in $\mathcal{R}$ within $V^{e^{\prime}}$ is at most

$$
\sum_{h=1}^{k} \lambda_{s+2}\left(t_{h}\right) \leq \lambda_{s+2}\left(\sum_{h=1}^{k} t_{h}\right)=\lambda_{s+2}\left(O\left(t \beta\left(\frac{t}{k}\right)\right)\right)=O\left(t \beta^{2}(t)\right) .
$$

Lemma 2.6. The total number of covered edge-crossings in $\mathcal{R}$, in the entire arrangement, is $O\left(k^{4} n^{2+\varepsilon}\right)$, for any $\varepsilon>0$.

Proof. Let $\mathcal{R}^{\prime}$ be the set of all covered edge-crossings in $\mathcal{R}$, but with certain multiplicities removed: For every four surfaces $\sigma_{1}, \sigma_{2}, \sigma_{3}, \sigma_{4} \in \Sigma$, we choose only one edge-crossing $\left(e, e^{\prime}, g\right)$ with $e \subseteq \sigma_{1} \cap \sigma_{2}$ and $e^{\prime} \subseteq \sigma_{3} \cap \sigma_{4}$ for $\mathcal{R}^{\prime}$, and, for every three surfaces $\sigma_{1}, \sigma_{2}, \sigma_{3} \in \Sigma$, we choose only one edge-crossing $\chi=\left(e, e^{\prime}, g\right)$ with $e \subseteq \sigma_{1} \cap \sigma_{2}$ and $\sigma(\chi)=\sigma_{3}$ for $\mathcal{R}^{\prime}$. Exploiting condition (R3) and the fact that there are at most $s$ edge-crossings defined by the same four surfaces, it is now sufficient to show that $\left|\mathcal{R}^{\prime}\right|=O\left(k^{3} n^{2+\varepsilon}\right)$, for any $\varepsilon>0$. 

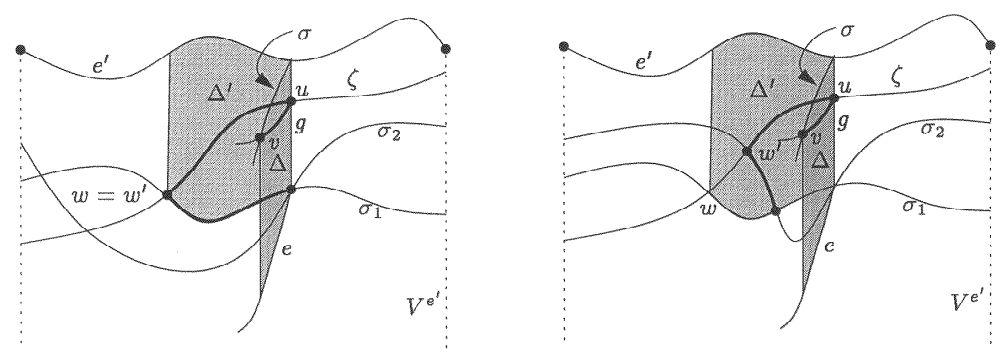

Fig. 8. Two cases of covered edge-crossings $\chi=\left(e, e^{\prime}, g\right) \in \mathcal{R}$, and the corresponding paths $\pi(\chi)$ (shown as fat curves).

Let $\chi=\left(e, e^{\prime}, g\right)$ be a covered edge-crossing in $\mathcal{R}^{\prime}$. Let $\sigma_{1}, \sigma_{2}$, and $\zeta \in \Sigma$ be as in the definition of a covered edge-crossing, and assume that $\sigma_{1}$ crosses $\zeta$ within $V^{e^{\prime}}$ to the left of $g$ at some point $w$; see Fig. 8. By condition (R4), there is an intersection point $v$ of $\zeta$ and $\sigma$ on $V_{e}$, where $\sigma=\sigma(\chi)$ is one of the two surfaces defining $e^{\prime}$, the portion of $\zeta \cap V_{e}$ between $v$ and $g$ does not meet $\sigma$, and the portion of $\sigma \cap V_{e}$ between $v$ and $g$ does not meet the other surface $\sigma^{\prime}$ incident to the top endpoint of $g$. Let $\Delta$ be the trapezoidal-like region within $V_{e}$, as defined in condition (R4), and let $\Delta^{\prime}$ be the trapezoidal-like region within $V^{e^{\prime}}$, as in the definition of covered edge-crossings. By the preceding arguments, at most $3 k$ surfaces cross either $\Delta$ or $\Delta^{\prime}$. See Fig. 8 for an illustration.

Let $K$ be a random sample of $n / k$ surfaces of $\Sigma$. As argued in [10], the probability that $K$ contains the five surfaces $\sigma_{1}, \sigma_{2}, \zeta, \sigma$, and $\sigma^{\prime}$, and does not contain any of the other surfaces crossing $\Delta \cup \Delta^{\prime}$, is at least $c / k^{5}$, for some absolute constant $c>0$. Let $\mathcal{R}_{K}^{\prime}$ be the set of edge-crossings $\chi \in \mathcal{R}^{\prime}$ that appear in $\mathcal{A}(K)$, in the sense that the above choice of surfaces in $K$ materializes. Since the expected size of $\mathcal{R}_{K}^{\prime}$ is at least $c / k^{5}$ times the size of $\mathcal{R}^{\prime}$, it suffices to prove that $\left|\mathcal{R}_{K}^{\prime}\right|=O\left(n^{2+\varepsilon} / k^{2}\right)$, for any $\varepsilon>0$.

So let $\mathcal{C}(K)=\mathcal{C}_{\omega}(K)$ denote the cell in $\mathcal{A}(K)$ that contains $\omega$, and consider an edge-crossing $\chi=\left(e, e^{\prime}, g\right) \in \mathcal{R}_{K}^{\prime}$. Clearly, the segment $g$ crosses in $\mathcal{A}(K)$ only the surface $\zeta$, and its portion below $\zeta$ is fully contained in $\mathcal{C}(K)$. For technical reasons, we distinguish between the two (top and bottom) sides of each surface in $\Sigma$; we appeal to the intuition of the reader, and refer to [3], [4], and [12] for a formal definition. An important property that this distinction has is that a curve drawn on the top side of a surface is not considered to cross a curve drawn on the bottom side.

We construct, for every $\chi \in \mathcal{R}_{K}^{\prime}$, a path $\pi=\pi(\chi)$ on $\partial \mathcal{C}(K)$ as the concatenation of the following three subpaths:

- $\pi_{1}(\chi)$ : the subarc of $\zeta \cap V_{e}$ connecting $v$ to $u=g \cap \zeta$;

- $\pi_{2}(\chi)$ : a subarc of $\zeta \cap V^{e^{\prime}}$ extending from $u$ toward $w$, stopping as soon as it hits either $\sigma_{1}$ or $\sigma_{2}$ in a point $w^{\prime}$ ( $w^{\prime}$ may or may not be equal to $w$, see the two cases in Fig. 8);

- $\pi_{3}(\chi)$ : this subpath extends from $w^{\prime}$ along $\sigma_{1} \cap V^{e^{\prime}}$ or $\sigma_{2} \cap V^{e^{\prime}}$ (depending on which surface contains $w^{\prime}$ ) toward $g$, and stops as soon as it hits the other surface defining $e$ (this can happen either at $g$ or earlier, see Fig. 8).

The first two portions $\pi_{1}(\chi)$ and $\pi_{2}(\chi)$ are drawn on the bottom side of $\zeta$, and the last portion $\pi_{3}(\chi)$ is drawn on the top side of $\sigma_{1}$ or $\sigma_{2}$; see Fig. 8. 
We draw a path $\pi(\chi)$ for each edge-crossing $\chi \in \mathcal{R}_{K}^{\prime}$.

Claim. The relative interiors of any two distinct paths $\pi(\chi), \pi\left(\chi^{*}\right)$ are disjoint.

Proof of Claim. Suppose to the contrary that $\pi(\chi)$ and $\pi\left(\chi^{*}\right)$ contain a common point, for two distinct edge-crossings $\chi=\left(e, e^{\prime}, g\right)$ and $\chi^{*}=\left(e^{*},\left(e^{\prime}\right)^{*}, g^{*}\right)$ in $\mathcal{R}_{K}^{\prime}$. Because of the top/bottom sidedness of the surfaces and the way in which the paths are drawn, only the following cases can occur.

$\pi_{1}(\chi)$ intersects $\pi_{1}\left(\chi^{*}\right)$ : By our construction, the path $\pi_{1}(\chi)$ lies directly above an edge of $\mathcal{A}(K)$ (the one containing $e$ ), starts (at its left endpoint) at an edge of $\mathcal{A}(K)$ and stops as soon as it passes directly below another edge (the edge $e^{\prime}$ ); a similar property holds for $\pi_{1}\left(\chi^{*}\right)$. It follows that if $\pi_{1}(\chi)$ and $\pi_{1}\left(\chi^{*}\right)$ have a common point (including even the sharing of an endpoint), then $e=e^{*}, e^{\prime}=\left(e^{\prime}\right)^{*}$, and the two paths must coincide completely. This implies that $\chi$ and $\chi^{*}$ are defined by the same four surfaces, which is a contradiction to the definition of $\mathcal{R}^{\prime}$.

$\pi_{2}(\chi)$ intersects $\pi_{2}\left(\chi^{*}\right)$ : The path $\pi_{2}(\chi)$ passes directly below an edge of $\mathcal{A}(K)$ (the one containing $e^{\prime}$ ), ends on an edge of $\mathcal{A}(K)$, and starts directly above an edge of $\mathcal{A}(K)$ (the one containing $e$ ). It may pass above other edges $\bar{e}$ of $\mathcal{A}(K)$, but then any such $\bar{e}$ is defined by the same two surfaces as $e$. It follows that if the relative interiors of $\pi_{2}(\chi)$ and $\pi_{2}\left(\chi^{*}\right)$ have a common point, then $e^{\prime}=\left(e^{\prime}\right)^{*}$, and hence $\sigma(\chi)=\sigma\left(\chi^{*}\right)$. Furthermore, the surfaces $\zeta$ on which these subpaths are drawn are the same, and $e$ and $e^{*}$ must be defined by the same two surfaces of $\Sigma$. Again, this is impossible by our definition of $\mathcal{R}^{\prime}$.

The case where the common point of $\pi_{2}(\chi)$ and $\pi_{2}\left(\chi^{*}\right)$ is an endpoint of, say, $\pi_{2}(\chi)$ is handled in a similar manner. That is, we note that if the surfaces $\zeta$ on which these subpaths are drawn are not the same, then the manner in which these subpaths are drawn on the bottom side of these surfaces is easily seen to imply that the paths do not meet at this point at all; see Fig. 9 for an illustration.

$\pi_{3}(\chi)$ intersects $\pi_{3}\left(\chi^{*}\right)$ : The path $\pi_{3}(\chi)$ lies below an edge of $\mathcal{A}(K)$ (the one containing $e^{\prime}$ ), and exactly one surface of $K$ (namely, $\zeta$ ) passes between the path and the edge. Hence, if the relative interiors of $\pi_{3}(\chi)$ and $\pi_{3}\left(\chi^{*}\right)$ have a common point, then $e^{\prime}=\left(e^{\prime}\right)^{*}$, and the surfaces on which these subpaths are drawn are the same. Hence $\pi_{3}(\chi)=\pi_{3}\left(\chi^{*}\right)$, since they both lie in $V^{e^{\prime}}$, on the same surface, and extend in both

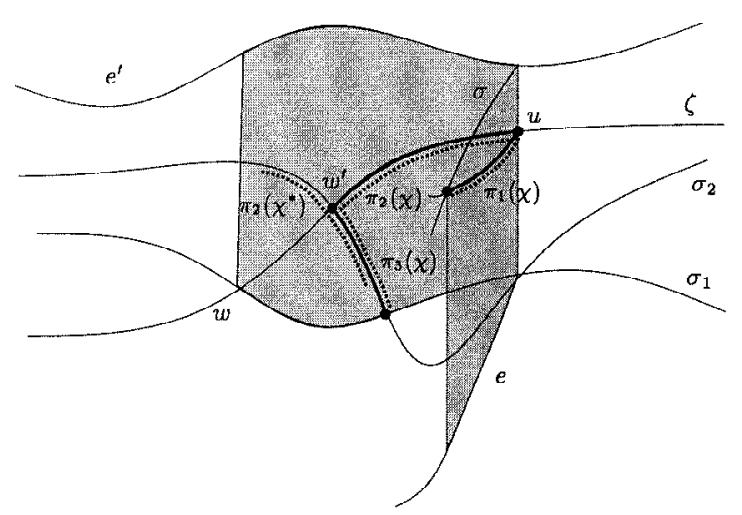

Fig. 9. The paths $\pi_{2}(\chi)$ and $\pi_{2}\left(\chi^{*}\right)$ have no point in common. 
directions until they hit an edge of $\mathcal{A}(K)$. This implies that $\chi$ and $\chi^{*}$ are again defined by the same four surfaces, which is impossible. Again, the case where the common point of $\pi_{3}(\chi)$ and $\pi_{3}\left(\chi^{*}\right)$ is an endpoint of one of these subpaths is handled as above.

Finally, $\pi_{1}(\chi)$ intersects $\pi_{2}\left(\chi^{*}\right)$ (or, symmetrically, $\pi_{1}\left(\chi^{*}\right)$ intersects $\pi_{2}(\chi)$ ): A point $p \in \pi_{2}\left(\chi^{*}\right)$ has to lie directly below an edge of $\mathcal{A}(K)$. The only point with this property on $\pi_{1}(\chi)$ is its endpoint $u$. On the other hand, $u$ lies directly above an edge of $\mathcal{A}(K)$. The only such point on $\pi_{2}\left(\chi^{*}\right)$ is its endpoint, and hence $e=e^{*}$ and $e^{\prime}=\left(e^{\prime}\right)^{*}$, which, as above, yields a contradiction.

This completes the proof of the claim.

We continue with the proof of the lemma. We now have a system $G$ of pairwise openly disjoint paths drawn on $\partial \mathcal{C}_{\omega}(K)$, and our next goal is to bound their number, using Euler's formula for planar graphs, in a manner similar to, though somewhat more complex than, the technique used by Tagansky [20]. This is done as follows.

Fix a face $f$ of $\partial \mathcal{C}_{\omega}(K)$ (which lies on either the top side or the bottom side of some surface), and clip all paths that cross $f$ to within $f$ (note that either all these clippings retain the first two portions of each such path, if $f$ lies on the bottom side of a surface, or they all retain the third portions of these paths, if $f$ lies on the top side). Let $G_{f}$ denote the resulting collection of clipped paths. We regard $G_{f}$ as a plane drawing of a graph, whose nodes are the edges of $f$ and whose arcs are the clipped paths. Since $f$ is (homeomorphic to) a planar region, we do indeed obtain a plane drawing of a planar graph, and we can apply Euler's formula to conclude that the number of arcs in $G_{f}$ is at most three times the number of edges of $f$, plus the number of faces of $G_{f}$ of degree 2. Applying this analysis to each (sided) face of $\mathcal{C}_{\omega}(K)$, and summing up these bounds, we conclude that the overall number of clipped subpaths is proportional to the complexity of $\mathcal{C}_{\omega}(K)$, which is $O\left((n / k)^{2+\varepsilon}\right)$, for any $\varepsilon>0$ [12], plus the overall number of graph-faces of degree 2 .

To get a better handle on those degree- 2 faces, we go over all faces $f$ of $\mathcal{C}_{\omega}(K)$, take each "run" of adjacent degree- 2 faces within $f$, and delete all their incident subpaths, except for the first and the last one. Clearly, the number of remaining subpaths is $O\left((n / k)^{2+\varepsilon}\right)$, for any $\varepsilon>0$.

Now take a full path $\pi(\chi)$. If either of its two clipped subpaths has survived after the above trimming, we charge $\pi(\chi)$ to that subpath. Since this charging is unique, the number of paths $\pi(\chi)$ of this kind is $O\left((n / k)^{2+\varepsilon}\right)$, for any $\varepsilon>0$. Suppose then that both clipped subpaths of $\pi(\chi)$ have been trimmed. This is easily seen to imply that there is a sequence of "parallel" paths, all of which connect between the same pair of edges of $\mathcal{C}_{\omega}(K)$, such that $\pi(\chi)$ is a middle element of the sequence. Recall that, in the notations used above, one of the terminal edges of $\pi(\chi)$ is incident to the two surfaces $\sigma_{1}, \sigma_{2}$ that meet also at the bottom endpoint of $g$ (recall that the endpoint of $\pi(\chi)$ need not coincide with the endpoint of $g$ ), and the other terminal edge is incident to $\zeta$ and to $\sigma(\chi)$. It follows that there are at least three different paths $\pi\left(\chi^{\prime}\right)$ in the above sequence, such that the corresponding edge-crossings $\chi^{\prime}$ share the three surfaces $\sigma_{1}, \sigma_{2}$, and $\sigma=\sigma\left(\chi^{\prime}\right)$. By our definition of $\mathcal{R}^{\prime}$, this is impossible, and therefore every path $\pi(\chi)$ is uniquely charged to one of its subpaths.

All these arguments readily imply that the total number of paths $\pi(\chi)$ that are drawn on $\partial \mathcal{C}_{\omega}(K)$ is $O\left((n / k)^{2+\varepsilon}\right)$, for any $\varepsilon>0$. This implies that $\left|\mathcal{R}_{K}^{\prime}\right|=O\left(n^{2+\varepsilon} / k^{2}\right)$, and, by our conclusions above, this completes the proof of the lemma. 
We now show how the bounds derived in Lemmas 2.5 and 2.6 imply our main result. Put $t^{*}=\sum_{e^{\prime}} t_{e^{\prime}}$, where the sum extends over all split edges $e^{\prime}$ at $\mathcal{C}$-level at most $k$, for which $t_{e^{\prime}}>4 k$. Lemmas 2.5 and 2.6 imply that

$$
|\mathcal{R}|=O\left(k^{4} n^{2+\varepsilon}\right)+\sum_{e^{\prime}} O\left(t_{e^{\prime}} \beta^{2}\left(t_{e^{\prime}}\right)\right)=O\left(k^{4} n^{2+\varepsilon}\right)+O\left(t^{*} \beta^{2}(n)\right) .
$$

On the other hand, for each edge $e^{\prime}$ as above, the number of vertices appearing in the first $3 k V$-levels of $\mathcal{A}\left(\Sigma^{e^{\prime}}\right)$ is $\Omega\left(t_{e^{\prime}} k\right)$. Indeed, the number of surfaces whose intersection arcs are fully contained within the first $3 k V$-levels of $\mathcal{A}\left(\Sigma^{e^{\prime}}\right)$ is at most $3 k$, because, for every such surface $\sigma$, the endpoints of the curve $\sigma \cap V^{e^{\prime}}$ must be among the first $3 k$ curves below each endpoint of $e^{\prime}$ (the curve cannot have an endpoint in the interior of $V^{e^{\prime}}$, because such an endpoint would have caused $e^{\prime}$ to be further split). Since $t_{e^{\prime}}>4 k$, at least $t_{e^{\prime}}-3 k>\frac{1}{4} t_{e^{\prime}}$ of these curves have a point at $V$-level $>3 k$, and thus each of them must contain at least $k$ vertices of $\mathcal{A}\left(\Sigma^{e^{\prime}}\right)$ at $V$-level $\leq 3 k$ (because, by definition, it also shows up among the top $2 k V$-levels). Since each such vertex induces an edge-crossing at $\mathcal{C}$-level at most $3 k$, it follows that

$$
C_{3 k}(\Sigma ; \omega)=\Omega\left(k \sum_{e^{\prime}} t_{e^{\prime}}\right)=\Omega\left(t^{*} k\right) .
$$

Hence, we have

$$
|\mathcal{R}|=O\left(k^{4} n^{2+\varepsilon}\right)+O\left(\frac{\beta^{2}(n)}{k} \cdot k t^{*}\right)=O\left(k^{4} n^{2+\varepsilon}\right)+O\left(\frac{\beta^{2}(n)}{k} \cdot C_{3 k}(n)\right) .
$$

Next, we estimate $C_{3 k}(n)$ by using the probabilistic technique of Clarkson and Shor [10], [16]. Since each edge-crossing $\left(e, e^{\prime}, g\right)$ is defined by four surfaces (two surfaces incident to $e$ and two incident to $e^{\prime}$ ), the Clarkson-Shor technique is easily seen to imply that $C_{3 k}(n)=O\left(k^{4} C_{0}(n / k)\right)$. Combining (3), (4), and the Clarkson-Shor bound, we readily obtain

$$
\begin{aligned}
C_{0}(n) & =O\left(\left(k \beta(k)+k^{3} \beta(n)\right) n^{2+\varepsilon}+k^{3} \beta(k) n \lambda_{s+2}\left(\frac{n}{k}\right)\right)+\frac{\beta^{3}(n)}{k^{2}} \cdot O\left(k^{4} C_{0}\left(\frac{n}{k}\right)\right) \\
& =O\left(k^{3} \beta(n) n^{2+\varepsilon}\right)+O\left(k^{2} \beta^{3}(n)\right) \cdot C_{0}\left(\frac{n}{k}\right) .
\end{aligned}
$$

The solution of this recurrence is $O\left(n^{2+\delta}\right)$, for any $\delta>\varepsilon$. This is shown by induction on $n$, choosing $k=\beta^{1+3 / \delta}(n)$ and using the fact that $\beta(n)$ is an extremely slowly growing function of $n$ (see also [1]).

In conclusion, we have thus obtained the main result of the paper:

Theorem 2.7. The complexity of the vertical decomposition of a single cell in an arrangement of $n$ algebraic surface patches in $\mathbb{R}^{3}$, such that the degrees of the surfaces and of their boundary curves are all bounded by some constant $b$, is $O\left(n^{2+\varepsilon}\right)$, for any $\varepsilon>0$, where the constant of proportionality depends on $\varepsilon$ and on $b$. 


\section{Applications}

\subsection{Constructing a Single Cell}

Theorem 3.1. Given an arrangement of $n$ algebraic surface patches in $\mathbb{R}^{3}$, such that the degrees of the surfaces and of their boundary curves are all bounded by some constant $b$, one can construct, in an appropriate model of computation, the cell of the arrangement containing a given point, in randomized expected time $O\left(n^{2+\varepsilon}\right)$, for any $\varepsilon>0$, where the constant of proportionality depends on $\varepsilon$ and on $b$.

Proof. As noted in the Introduction, this can be accomplished, in a rather routine manner, by applying the lazy randomized incremental framework of de Berg et al. [6]. We content ourselves with a brief sketch. The model of computation that we assume is that of exact rational arithmetic, using one which can perform any primitive algebraic operation involving a constant number of surfaces (such as intersecting three surfaces, computing edge-crossings induced by four surfaces, etc.) in constant time.

The algorithm maintains the vertical decomposition of a collection of cells of the arrangement while adding the surface patches one by one in a randomly chosen order. The collection of cells is guaranteed to contain the cell we are interested in. In general it will include some other cells as well, since it seems to be too hard to determine whether a new surface patch splits the current single cell or not.

Recall that vertical decomposition is defined in two stages: In the first stage, we erect primary walls from edges of the arrangement, and obtain cylindrical cells whose bottom and top facet are both contained in a single surface patch and have the same $x y$-projection. In the second stage, we partition these cylinders into constant-complexity "prisms," using secondary walls defined by a trapezoidal decomposition in the $x y$-projection. We call the final resulting prisms boxes.

As an auxiliary structure, our algorithm also maintains a history graph as introduced by Boissonnat et al. [7]. The history graph is a rooted, directed, acyclic graph whose nodes correspond to boxes of the vertical decomposition at some intermediate stage. The leaf nodes of the graph are colored either green or red. The green leaf nodes correspond to the boxes of the vertical decomposition of the currently maintained collection of cells. We also maintain a graph on the green leaf nodes that will allow us to transverse the maintained cells of the current vertical decomposition.

To insert a new surface $\sigma$, we first have to find all green leaf nodes intersected by $\sigma$. This is done by a graph traversal of the history graph, starting at the root. This requires that we can test whether $\sigma$ intersects a given box. Since boxes are defined by a constant number of polynomials, this is a constant time operation in our model.

Every green leaf box $\tau$ intersected by $\sigma$ is split into a constant number of new boxes. A new green leaf is created for every new box, and it is linked to $\tau$. The adjacency relations are updated at the same time.

The new set of boxes will not, in general, be a proper vertical decomposition of the new surface arrangement. In the next step, we have to merge boxes that are separated by secondary wall sections that are no longer part of the vertical decomposition. This is relatively easy for boxes whose top and bottom facets are distinct from $\sigma$.

To correct the situation for boxes whose top or bottom facet is $\sigma$, we recompute the 
two-dimensional trapezoidal decomposition for all relevant facets on the surface of $\sigma$. A secondary history graph is created to aid traversing the history graph for these boxes, see [6] for details.

After inserting surface patch number $2^{i}$, for $1 \leq i<\log n$, we identify the cell containing the marking point. This is done by traversing the vertical decomposition, starting from the box that contains the given point. All boxes of the current set that do not lie inside the cell are colored red, and will therefore not be subdivided any further.

The stated bound on the running time of the algorithm follows immediately from the general results on lazy randomized incremental construction [6], once we plug in our bound on the complexity of the vertical decomposition of a single cell.

\subsection{Motion Planning for Systems with Three Degrees of Freedom}

As noted in the Introduction, the main application of Theorems 2.7 and 3.1 is to motion planning for arbitrary systems with three degrees of freedom. This application is described in detail in the survey papers [13] and [18], see also [3]. The results of our paper imply the following:

Theorem 3.2. Let $B$ be a robot system with three degrees offreedom, such that the free configuration space of $B$ can be described as a Boolean combination of $n$ polynomial equalities and inequalities, of constant maximum degree $b$, in the three parameters that define the degrees of freedom of $B$. Then, given any two free placements $Z_{1}, Z_{2}$ of $B$, it can be determined, in randomized expected time $O\left(n^{2+\varepsilon}\right)$, for any $\varepsilon>0$, whether there exists a collision-free motion of $B$ from $Z_{1}$ to $Z_{2}$, and, if so, produce such a motion. (The constant of proportionality in this bound depends on $\varepsilon$ and on $b$.)

As mentioned in the Introduction, this settles a long-standing open problem, which was previously solved only for some special systems.

\section{Acknowledgments}

We wish to thank Danny Halperin for useful discussions concerning the problems studied in this paper. Part of the work on the paper has been carried out in the Mathematical Research Institute of Tel Aviv University, which the first author visited in the spring of 1995.

\section{References}

1. P.K. Agarwal, O. Schwarzkopf, and M. Sharir, The overlay of lower envelopes in three dimensions and its applications, Discrete Comput. Geom. 15 (1996), 1-13.

2. P.K. Agarwal, M. Sharir, and P. Shor, Sharp upper and lower bounds for the length of general DavenportSchinzel sequences, J. Combin. Theory Ser. A. 52 (1989), 228-274.

3. B. Aronov and M. Sharir, Triangles in space, or: Building (and analyzing) castles in the air, Combinatorica 10(2) (1990), 137-173. 
4. B. Aronov and M. Sharir, Castles in the air revisited, Discrete Comput. Geom. 12 (1994), 119-150.

5. B. Aronov and M. Sharir, On translational motion planning in three dimensions, Proc. 10th ACM Symp. on Computational Geometry, 1994, pp. 21-30. Also to appear in SIAM J. Comput.

6. M. de Berg, K. Dobrindt, and O. Schwarzkopf, On lazy randomized incremental construction, Discrete Comput. Geom. 14 (1995), 261-286.

7. J.-D. Boissonnat, O. Devillers, R. Schott, M. Teillaud, and M. Yvinec, Applications of random sampling to on-line algorithms in computational geometry, Discrete Comput. Geom. 8 (1992), 51-71.

8. B. Chazelle, H. Edelsbrunner, L. Guibas, and M. Sharir, A singly exponential stratification scheme for real semi-algebraic varieties and its applications, Proc. 16th Internat. Colloq. on Automata, Languages and Programming, 1989, pp. 179-193. (Also in Theoretical Computer Science 84 (1991), 77-105.)

9. K. Clarkson, H. Edelsbrunner, L. Guibas, M. Sharir, and E. Welzl, Combinatorial complexity bounds for arrangements of curves and spheres, Discrete Comput. Geom. 5 (1990), 99-160.

10. K. Clarkson and P. Shor, Applications of random sampling in computational geometry, II, Discrete Comput. Geom. 4 (1989), 387-421.

11. D. Halperin and M. Sharir, New bounds for lower envelopes in three dimensions, with applications to visibility in terrains, Discrete Comput. Geom. 12 (1994), 313-326.

12. D. Halperin and M. Sharir, Almost tight upper bounds for the single cell and zone problems in three dimensions, Discrete Comput. Geom. 14 (1995), 385-410.

13. D. Halperin and M. Sharir, Arrangements and their applications in robotics: Recent developments, in The Algorithmic Foundations of Robotics (K. Goldberg, D. Halperin, J.C. Latombe, and R. Wilson, Eds.), A.K. Peters, Boston, MA, 1995, pp. 495-511.

14. D. Halperin and M. Sharir, A near-quadratic algorithm for planning the motion of a polygon in a polygonal environment, Discrete Comput. Geom. 16 (1996), 121-134.

15. S. Hart and M. Sharir, Nonlinearity of Davenport-Schinzel sequences and of generalized path compression schemes, Combinatorica 6 (1986), 151-177.

16. M. Sharir, On $k$-sets in arrangements of curves and surfaces, Discrete Comput. Geom. 6 (1991), $593-613$.

17. M. Sharir, Almost tight upper bounds for lower envelopes in higher dimensions, Discrete Comput. Geom. 12 (1994), 327-345.

18. M. Sharir, Arrangements in higher dimensions: Voronoi diagrams, motion planning, and other applications, Proc. 4th Internat. Workshop on Algorithms and Data Structures (WADS'95), Lecture Notes in Computer Science, Vol. 955, Springer-Verlag, Berlin, 1995, pp. 109-121.

19. M. Sharir and P. Agarwal, Davenport-Schinzel Sequences and Their Geometric Applications, Cambridge University Press, New York, 1995.

20. B. Tagansky, A new technique for analyzing substructures in arrangements, Discrete Comput. Geom. 16 (1996), 455-479.

Received May 30, 1996, and in revised form February 18, 1997. 